\title{
On-Chip Cell-Cell Interaction Monitoring at Single-Cell Level by Efficient Immobilization of Multiple Cells in Adjustable Quantities
}

\author{
Xiaoqing Tang, ${ }^{\dagger}$ Xiaoming Liu,,${ }^{\dagger}$ Pengyun Li,${ }^{\dagger}$ Fengyu Liu,${ }^{\dagger}$ Masaru Kojima,,${ }^{\ddagger}$ Qiang Huang ${ }^{\dagger}$ and \\ Tatsuo Arai ${ }^{\dagger, \S}$ \\ †Key Laboratory of Biomimetic Robots and Systems, Ministry of Education, State Key Laboratory of Intelligent Control \\ and Decision of Complex System, Beijing Advanced Innovation Center for Intelligent Robots and Systems, and School of \\ Mechatronical Engineering, Beijing Institute of Technology, Beijing 100081, China. \\ *Department of Materials Engineering Science, Osaka University, Osaka 560-8531, Japan.
}

§Department of Mechanical and Intelligent Systems Engineering, the University of Electro-Communications, Tokyo 1828585, Japan.

*Phone: +8615101117324; E-mail: liuxiaoming555@bit.edu.cn.

\section{Table of Contents}

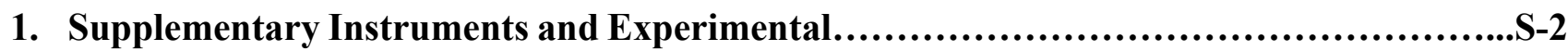

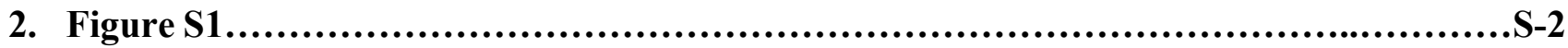

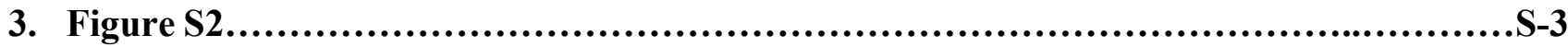

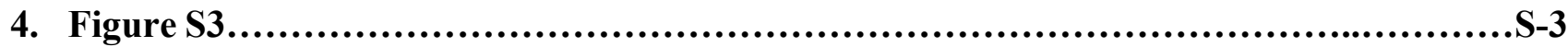

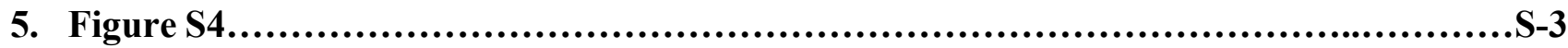

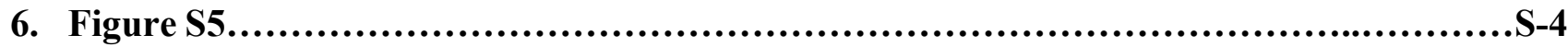

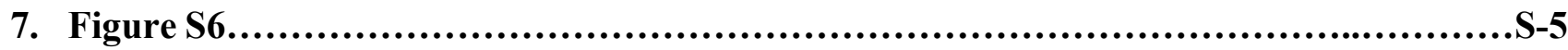

8. Supplementary Cell Viability Analysis................................................

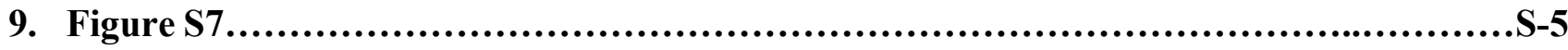




\section{Supplementary Instruments and Experimental}

Experimental system set-up. The cell suspension was flowed through the fabricated microfluidic chip by syringe pumps (LSP02-1B) with $1 \mathrm{ml}$ syringes and the whole manuscript system was set up under an inverted microscope (Olympus IX73) connected to a camera (DFK 23U274). The experimental system set-up was shown in Figure S1.

Fabrication of the Microfluidic Chips. To fabricate the microfluidic chips, standard photolithography with SU8 photoresist was used to create the two-layer mold, including a $3 \mu \mathrm{m}$-thick SU8-1050 layer for forming gap of the capture units and a $16 \mu \mathrm{m}$ thick SU8-1070 layer for shaping the main channel according to the design, followed by soft lithography to create PDMS molds. The fabrication was carried out as following: SU8-1050 photoresist (Switzerland Gersteltec) was spin-coated on a silicon wafer at a rotation speed of $3000 \mathrm{rpm}$ for $40 \mathrm{~s}$ to form the first layer. Then, the wafer was exposed to UV light for a short period (1.1 s) in a vacuum. After UV exposure, the wafer was pre-baked $\left(6 \mathrm{~min}, 95^{\circ} \mathrm{C}\right)$. Excess SU8 was removed by submersion in a developer solution $(15 \mathrm{~s})$. The hard bake was conducted under conditions $\left(7 \mathrm{~min}, 150^{\circ} \mathrm{C}\right)$ optimized to cure the first pattern while preventing cracking.

After the first layer was patterned, the second layer of SU8-1070 photoresist was spin-coated on top of the first and $5000 \mathrm{rpm}$ for $60 \mathrm{~s}$. Unlike with the first layer, a relaxation step $(>1 \mathrm{~h})$ and longer pre-bake $\left(65^{\circ} \mathrm{C}, 15\right.$ min followed by $\left.95^{\circ} \mathrm{C}, 35 \mathrm{~min}\right)$ were carried out. Then, the photomask was aligned with the pattern of the first layer using the alignment marks, and the wafer was exposed to UV light $(10 \mathrm{~s})$. A post-exposure bake $\left(45 \mathrm{~min}, 80^{\circ} \mathrm{C}\right)$, development $(170-180 \mathrm{~s})$, and hard bake $\left(2 \mathrm{~h}, 150^{\circ} \mathrm{C}\right)$ were carried out to finalize the mold.

For soft lithography, PDMS was poured over the SU8 mold and baked $\left(2 \mathrm{~h}, 65^{\circ} \mathrm{C}\right)$ to cure the final structure. After the PDMS had fully cured, each device was cut from the PDMS and holes were punched as the inlet and outlet. Then, the PDMS was bonded to a glass slide by oxygen plasma surface treatment, yielding a completed microfluidic chip (Figure S2).

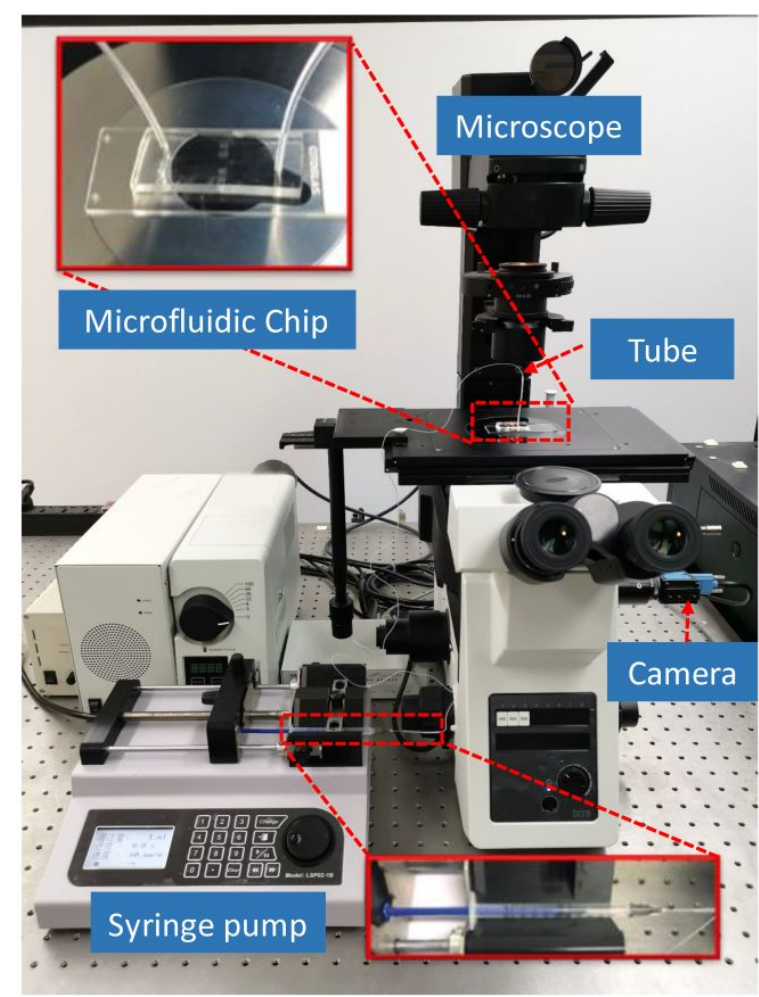

Figure S1. Experimental system set-up. 


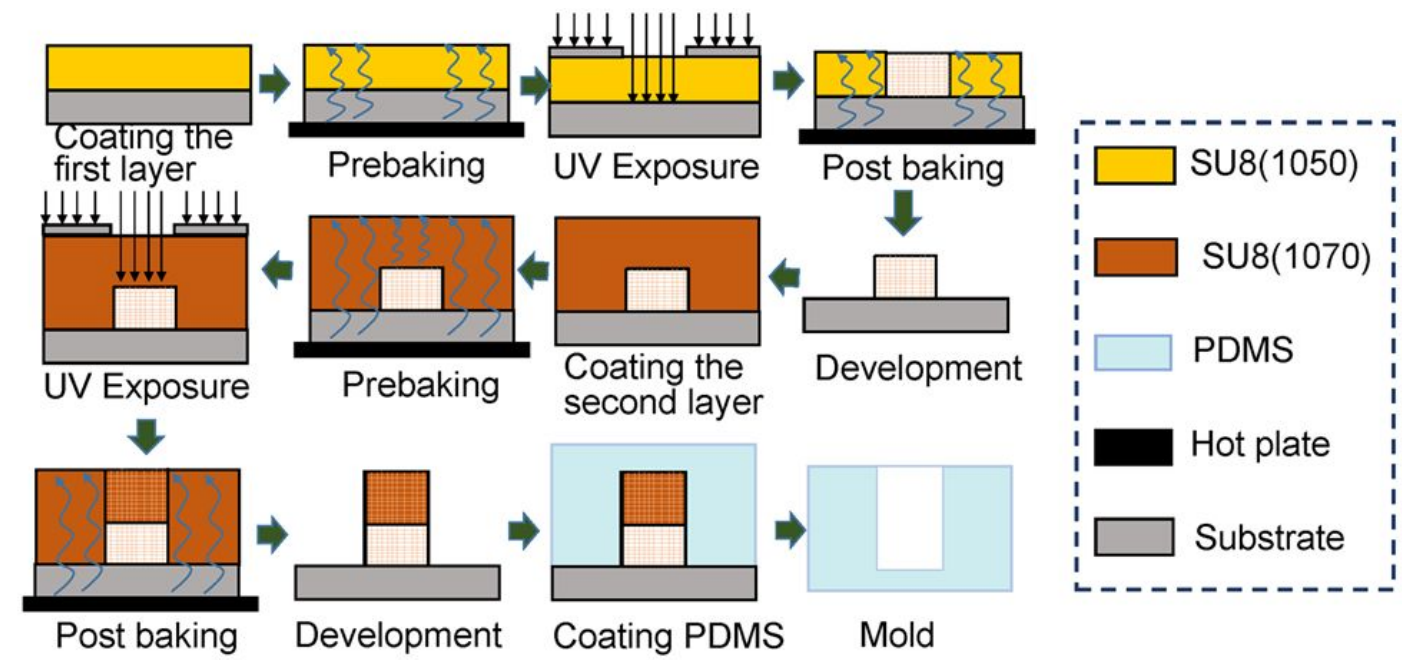

Figure S2. Fabrication of the proposed microfluidic chip

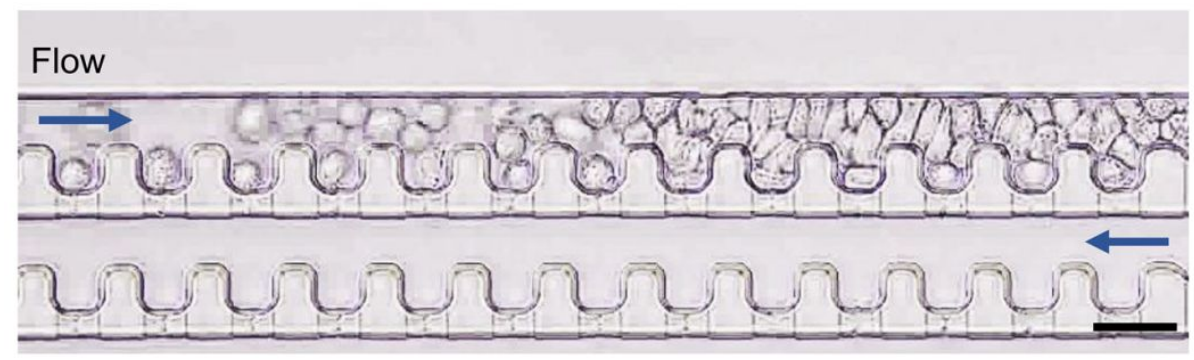

Figure S3. Cell aggregation in the main channel of the device when $W_{m}=15 \mu \mathrm{m}, L_{g}=10 \mu \mathrm{m}$. Scale bar is $30 \mu \mathrm{m}$.

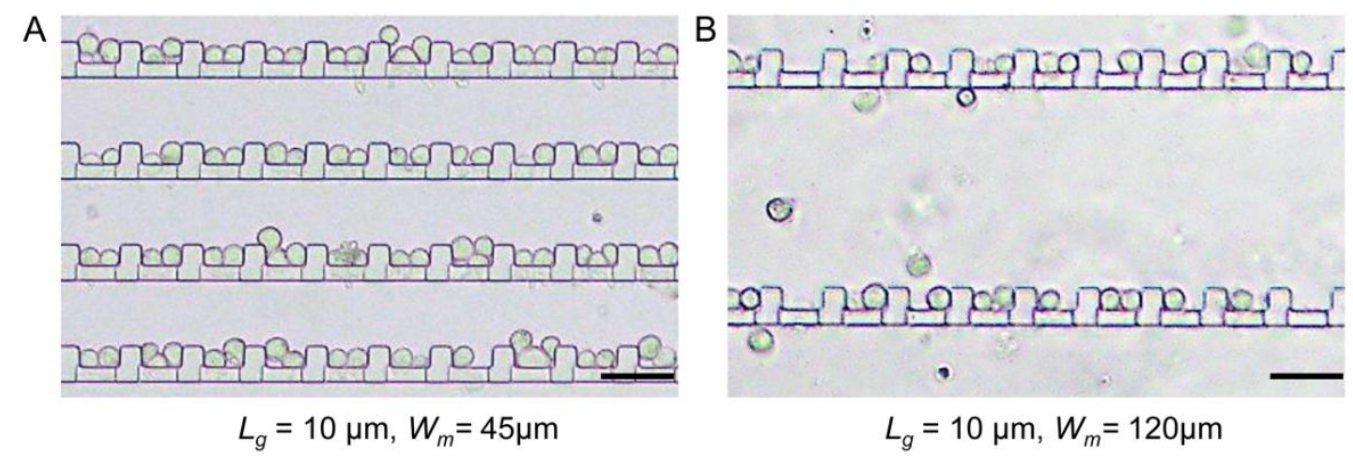

Figure S4. Representative bright-field images of trapping more/less than two cells in per capture unit of the microfluidic chips aiming to capture two cells under various $W_{m}$ dimensions $\left(L_{g}=10 \mu \mathrm{m}\right)$ : A) there were more than two cells in a single capture unit, when $W_{m}=$ $45 \mu \mathrm{m}, \mathrm{B})$ there was one cell or no cells in a single capture unit, when $W_{m}=120 \mu \mathrm{m}$. Scale bars are $50 \mu \mathrm{m}$. 
A

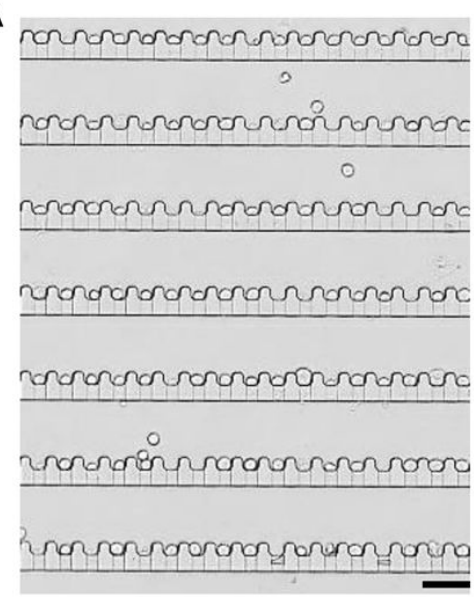

One cell $\left(L_{g}=20 \mu \mathrm{m}, W_{m}=60 \mu \mathrm{m}\right.$,

D TSR=95\%)

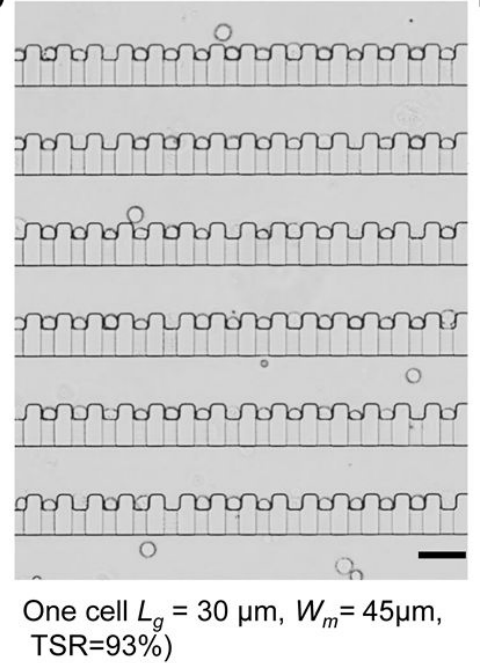

B

momormanaming

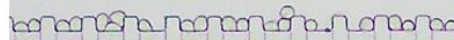

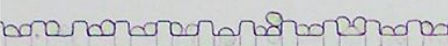

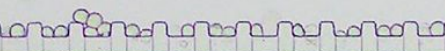

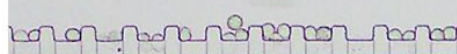

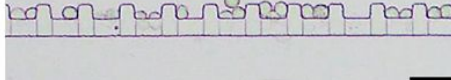

Two cells $\left(L_{g}=20 \mu \mathrm{m}, W_{m}=90 \mu \mathrm{m}\right.$,

E

TSR $=82 \%$ )

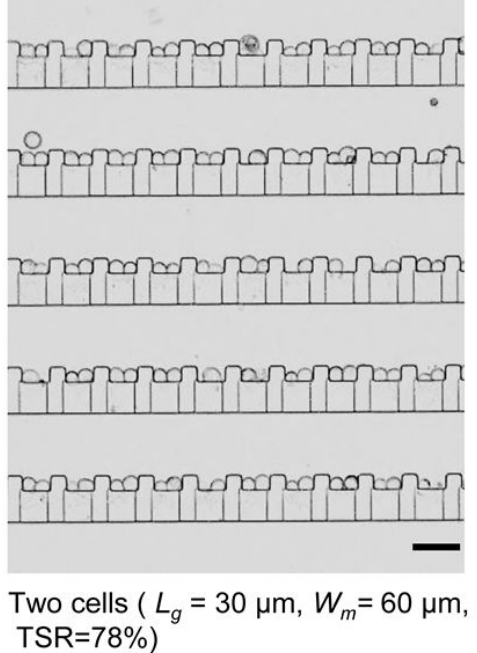

C

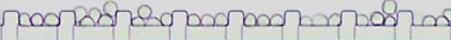

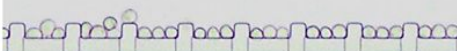

00

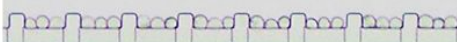
(1)

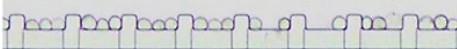

o

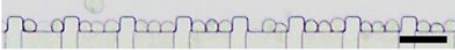

Three cells $\left(L_{g}=20 \mu \mathrm{m}, W_{m}=105 \mu \mathrm{m}\right.$ $\mathrm{F}^{\mathrm{TSR}}=78 \%$ )
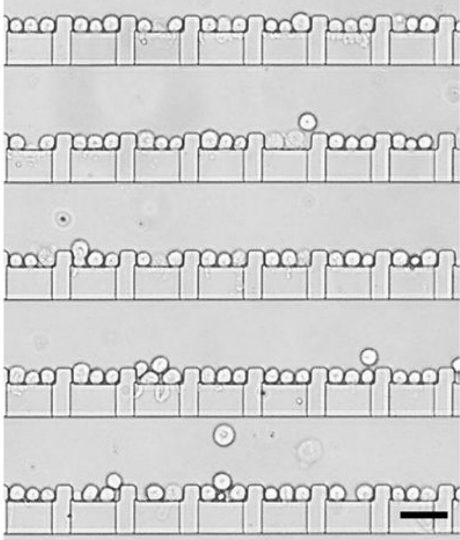

Three cells $\left(L_{g}=30 \mu \mathrm{m}, W_{m}=75 \mu \mathrm{m}\right.$ TSR $=75 \%$ )

Figure S5. Bright-field images of optimal trapping in microfluidic devices designed to capture one cell per capture unit when A) $L_{g}=$ $20 \mu \mathrm{m}$ and D) $L_{g}=30 \mu \mathrm{m}$, two cells per capture unit when B) $L_{g}=20 \mu \mathrm{m}$ and E) $L_{g}=30 \mu \mathrm{m}$, and three cells per capture unit when C) $L_{g}=20 \mu \mathrm{m}$ and F) $L_{g}=30 \mu \mathrm{m}$. The other parameters of the microfluidic device were the same as those used in the simulation shown in Figure 3. Scale bars are $50 \mu \mathrm{m}$. 

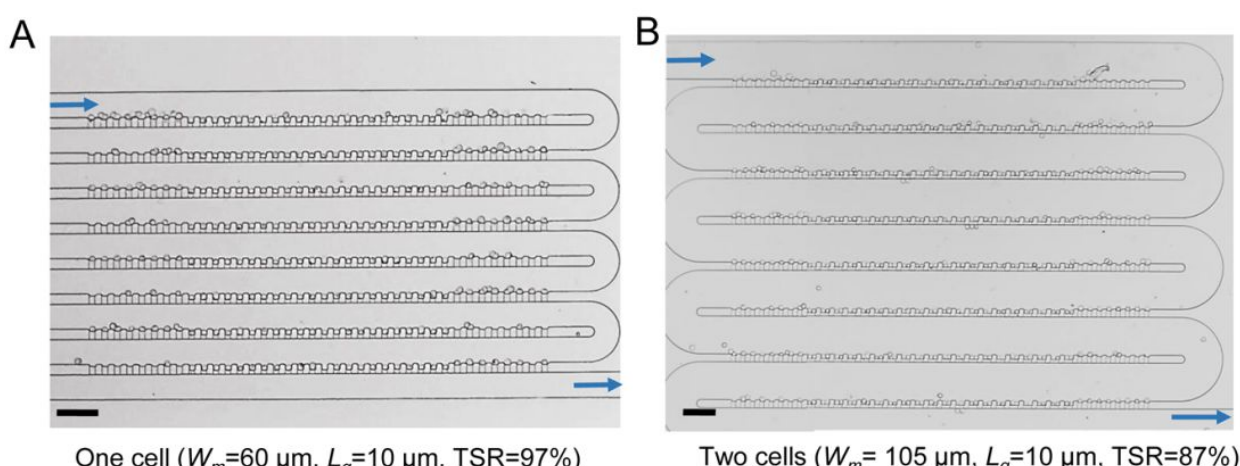

One cell $\left(W_{m}=60 \mu \mathrm{m}, L_{g}=10 \mu \mathrm{m}, \mathrm{TSR}=97 \%\right)$
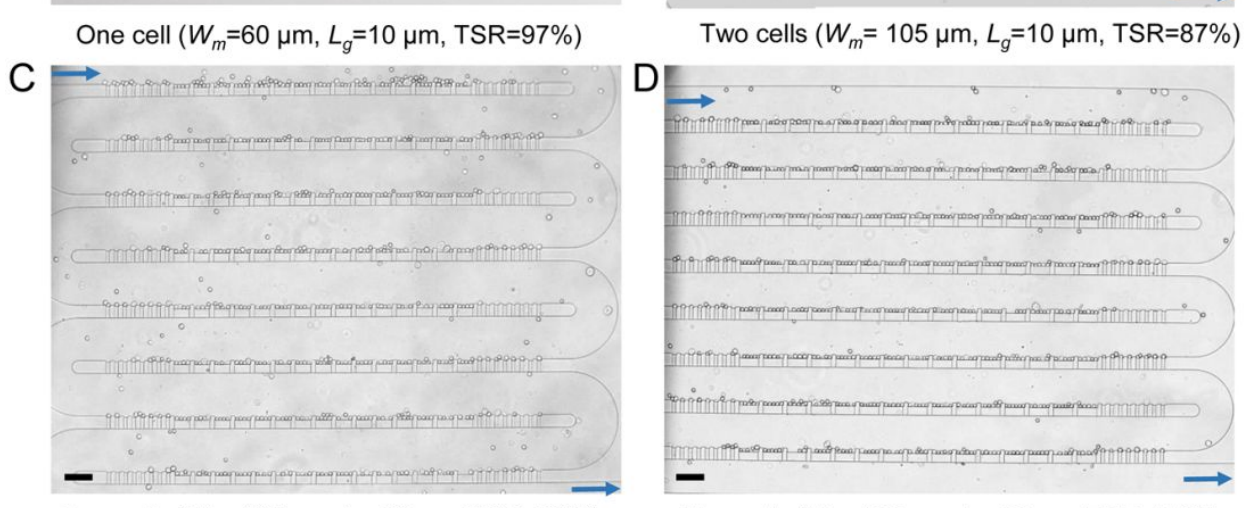

Four cells $\left(W_{m}=135 \mu \mathrm{m}, L_{g}=30 \mu \mathrm{m}, \mathrm{TSR}=58 \%\right)$

Five cells $\left(W_{m}=120 \mu \mathrm{m}, L_{g}=30 \mu \mathrm{m}, \mathrm{TSR}=54 \%\right)$

Figure S6. The overall bright-field images of cell trapping in microfluidic devices designed to capture A) single cell per capture unit $\left(W_{m}=60 \mu \mathrm{m}, L_{g}=10 \mu \mathrm{m}, \mathrm{TSR}=97 \%\right)$, B) two cells per capture unit $\left(W_{m}=105 \mu \mathrm{m}, L_{g}=10 \mu \mathrm{m}, \mathrm{TSR}=87 \%\right)$, C) four cells per capture unit $\left(W_{m}=135 \mu \mathrm{m}, L_{g}=30 \mu \mathrm{m}, \mathrm{TSR}=58 \%\right)$, and D) five cells per capture unit $\left(W_{m}=120 \mu \mathrm{m}, L_{g}=30 \mu \mathrm{m}\right.$, TSR=54\%). The other parameters of the microfluidic device were the same as those used in the simulation shown in Figure 3. Scale bars are $100 \mu \mathrm{m}$.

\section{Supplementary Cell Viability Analysis}

For testing biological compatibility of the microfluidic device, we did the statistical experiments on the survival rate of cells in the trapping arrays. The cells were suspended in PBS and dyed by incubating them in fluorochrome calcein-AM $(2 \mu \mathrm{M}$; CA1630 Solarbio) and PI (4 $\mu \mathrm{M}$; P8080 Solarbio) for $15 \mathrm{~min}$ in an incubator and were washed two times with fresh PBS. The stained cell suspension was flowed into the trapping arrays and then the cell viability was measured in the microfluidic device at 5-hour intervals for 25 hours.

The statistic results indicated that the proposed microfluidic device has a good biological compatibility (Figure S7).

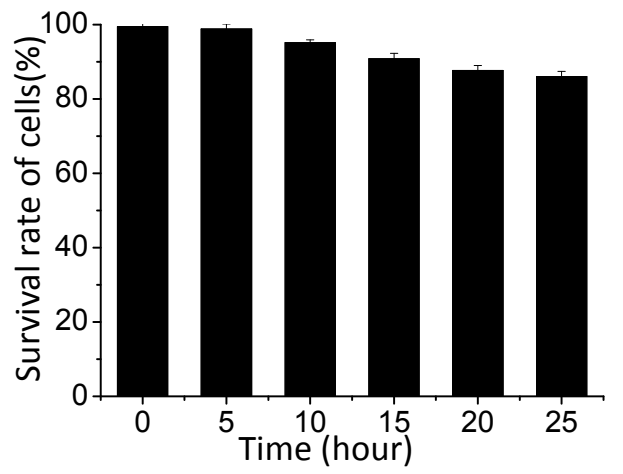

Figure S7. Cell viability in the proposed microfluidic device. 